\title{
CALCUlating Cost Distributions OF A MULTISERVICE LOSS SYSTEM
}

\author{
Jorma Jormakka \\ Department of Communications and Networking, Aalto University, Espoo, Finland \\ Sourangshu Ghosh \\ Department of Civil Engineering, Indian Institute of Technology Kharagpur, Kharagpur, West Bengal, India
}

\begin{abstract}
Congestion pricing has received lots of attention in the scientific discussion. Congestion pricing means that the operator increases prices at the time of congestion and the traffic demand is expected to decrease. In a certain sense, shadow prices are an optimal way of congestion pricing: users are charged shadow prices, i.e., the expectations of future losses because of blocked connections. The shadow prices can be calculated exactly from Howard's equation, but this method is difficult. The paper presents simple approximations to the solution of Howard's equation and a way to derive more exact approximations. If users do not react by lowering their demand, they will receive higher bills to pay. Many users do not react to increased prices but would want to know how the congestion pricing mechanism affects the bills. The distribution of the price of a connection follows from knowing the shadow prices and the probability of a congestion state. There is another interesting distribution. The network produces profit to the operator, or equivalently, blocked connections produce a cost to the operator. The average cost rate can be calculated from Howard's equation, but the costs have some distribution. The distribution gives the risk that the actual costs exceed the average costs, and the operator should include this risk to the prices. The main result of this paper shows how to calculate the distribution of the costs in the future for congestion pricing by shadow prices and for congestion pricing with a more simple pricing scheme that produces the same average costs.
\end{abstract}

Index Terms- Pricing, loss networks, Markov decision processes, blocking probability.

\section{INTRODUCTION}

$\mathrm{C}$ ONGESTION pricing is a state-dependent pricing strategy where the prices are higher when the network is congested. Congestion pricing has been studied extensively as a congestion control mechanism for elastic traffic demand: if users react to increased prices by lowering their demand, then the operator can use prices as a congestion control mechanism. Most authors consider congestion pricing of elastic traffic using the Game Theory [1], [2], [3], [4], and [5]. Shadow prices turn out to be the congestion prices that optimize the utility functions of the operator and the users.

The main focus in this paper is in distributions of costs when congestion pricing is applied. For a business user the interesting question is the cost of a connection. The bills of the business users are followed up and the company tries to notice errors in billing by the operator, abuse of the connections in some way, and malfunctioning of equipment, all of which can be the reason for exceptionally high bills. If a user comes to the network at a random time without knowing the congestion stage of the network and if the operator uses congestion pricing, then he will receive variable bills for the same service. Thus, congestion pricing is one cause to variability of the bill and both the company and the operator should know the effect of this mechanism. The expected distribution of the cost of a connection is obtained from the average relative costs and the state probability. Average relative costs have been calculated by many authors, the closest to this work are the calculations in [7], [10], [11] and [12]. The presented paper gives a simple approximate solution that does not require matching of parameters and shows the shape of the cost function better. A series form solution for deriving better approximation is also given.

It is important for an operator using shadow prices to know how much variability there can be in the costs over a longer time period. This is not the same as variability of the shadow prices. It is a question of the limit cost distribution. The main contribution of the paper is calculation of the cost distribution. Cost distributions have not been calculated before in the literature to Author's knowledge. Calculating the cost distribution for shadow prices turns out to be difficult, but a simpler pricing scheme can be created. For a user and the operator this simpler scheme gives practically the same prices and costs as shadow prices, but the cost distribution can be expressed by a closed formula.

We will not assume that the majority of users respond to congestion prices by lowering their traffic demand. There are reasons for dropping this assumption. Firstly, the users must notice that prices have been increased. Many users are a bit lazy in reacting to information of this type, so probably there should be an automatic agent in the user's terminal but that is unlikely to happen in the near future. Secondly, the user should want to reduce his demand. If there is an alternative network available the user may try to switch to the alternative connection. Often the user is connected to one operator's network only and cannot easily respond to price increases by shifting traffic to another network. In such a situation he should reduce his traffic demand. The need for congestion control is mainly from real-time services, today voice calls and in the future multimedia calls. The peak traffic of these 
services has traditionally been in the business days in the busy periods. There is no reason to expect that this situation would essentially change in the future. Bills of business users are paid by the company and both the users and the company are in the opinion that business time is so valuable that operator bills should not interfere with the ability to communicate when needed. It is unlikely that business users will respond to increased prices by lowering their demand. Some other users may indeed lower their demand and call blocking may be reduced or quality of service is improved by this method therefore an operator may want to use congestion pricing. For a business user the main visible effect of congestion pricing is the variability of the cost of a connection.

Following [6] we treat the system as a multiservice loss system since the future applications where congestion pricing could be appropriate are most probably multimedia calls or similar services. While packet networks are queuing systems, many services still require sufficient effective bandwidth and treating the network as a loss system often gives a fairly good approximation also for packet networks. Thus, we will not study congestion pricing as a congestion control mechanism of elastic traffic in a packet network. The setting is more similar to that in [6], where possibly state-dependent prices are used in a multiservice loss network, or [7] presenting a method to calculate shadow prices a multiservice loss network.

The paper is organized as follows. In Section II multiservice loss system is described. Section III $A$. shows how to calculate average costs using shadow prices. This calculation also gives the distribution of costs of connections in a user's bill. Section III $B$. presents cost distributions to an operator over a longer time if shadow prices are strictly followed. The results are complicated and solution can be given only to one simple blocking policy. Section IV has the main result of the paper: an exact solution to the cost distribution for a charging policy, which gives the same average costs as shadow prices and for a user is practically identical.

\section{MultiservicE LOSS SYSTEM}

A multiservice loss system (see e.g [8]) has a number of different connection types indexed by $j \in\{1, \ldots, K\}$. Each has own bandwidth demand: a call of type $j$ needs $b_{j}$ units of capacity. Each call type has a Poisson arrival process with an arrival rate $\lambda_{j}$. The call holding time for each call type is negatively exponentially distributed with the service rate $\mu_{j}$. Let the set $\Omega$ be the set of admitted state vectors $\bar{q}$. The policy sets $R_{\bar{q}}=\left\{j \mid \bar{q}+e_{j} \in \Omega\right\}$ define whether a call is accepted. Here $\bar{q}+e_{j}=\left(q_{1}, \ldots q_{j}+1, \ldots q_{K}\right)$ where $e_{j}$ has 1 in the index $j$, i.e. $e_{j}=(0, \ldots, 1, \ldots)$. The policy "a new call of type $j$ is accepted in the state $\bar{q}$ " is expressed using policy sets with the condition $j \in R_{\bar{q}}$.
Example 1. A call of type $k$ takes $d_{k}$ capacity units and the maximum number of capacity units is $C$. If the total number of used capacity units

$$
c=\sum_{k=1}^{K} d_{k} q_{k}=\bar{q}^{T} \bar{d} \text { is larger than } C-d_{k},
$$

a call of type $k$ is blocked. Otherwise it is accepted. Thus

$$
R_{\bar{q}}=\left\{j \mid \bar{q}^{T} \bar{d} \leq C-d_{j}\right\} .
$$

Example 2: A call of type $k$ takes $d_{k}$ capacity units. If the number of used capacity units by calls of type $k$ exceeds a threshold $C_{k}$ then a call of type $k$ is blocked.

The policy in Example 2 is less efficient than the policy in Example 1 because not all capacity is available to all call types.

The instantaneous transition matrix for the multi-service loss system can be written down by looking at the system at the state $\bar{q}=\left(q_{1}, \ldots, q_{K}\right)$ on a moment of time $n$. Let the total time be $T$ and let there be $N$ discrete time steps in time $T$. (We let $N \rightarrow \infty$.) Then we have the following transitions:

- a connection of type $j$ is accepted $(n, \bar{q}) \rightarrow\left(n+1, \bar{q}+e_{j}\right)$ with probability $\frac{T}{N} \lambda_{j} 1_{j \in R_{\bar{q}}}$,

- the state does not change $(n, \bar{q}) \rightarrow(n+1, \bar{q})$ with probability $1-\frac{T}{N} \sum_{j \in R_{\dot{q}}} \lambda_{j}-\frac{T}{N} \sum_{j=1}^{K} \mu_{j} q_{j}$

- a connection of type $j$ finishes $(n, \bar{q}) \rightarrow\left(n+1, \bar{q}-e_{j}\right)$ with probability $\frac{T}{N} \mu_{j} q_{j}$.

These probabilities give the instantaneous transition matrix $\mathbf{Q}$ which we express in the component form. Let $s_{n, q_{1}, \ldots, q_{K}}$ be the probability of the state $\left(q_{1}, \ldots, q_{K}\right)$ at the time $n$. The transition equations of a multiservice loss system have the following form:

$$
\begin{aligned}
& s_{n+1, q_{1}, \ldots, q_{K}}=s_{n, q_{1}, \ldots, q_{K}}-\sum_{j=1}^{K} \frac{\lambda_{j} T}{N} s_{n, q_{1}, \ldots, q_{K}} 1_{j \in R_{\bar{q}}} \\
& -\sum_{j=1}^{K} \frac{\mu_{j} T}{N} q_{j} s_{n, q_{1}, \ldots, q_{K}}+\sum_{j=1}^{K} \frac{\lambda_{j} T}{N} s_{n, q_{1}, \ldots, q_{j}-1, \ldots, q_{K}} 1_{j \in R_{\bar{q}-e_{j}}} \\
& \sum_{\bar{q} \in \Omega} s_{n, q_{1}, \ldots, q_{K}}=1,0 \leq s_{n, q_{1}, \ldots, q_{K}} \leq 1 .
\end{aligned}
$$

Remark 1. For the policies in Examples 1 and 2 the recursion equations (2) satisfy for every $j$ the condition

$$
s_{n, q_{1}, \ldots, q_{j}+1, \ldots q_{K}}=0 \quad \text { if } j \notin R_{\bar{q}} .
$$


This is so because if $j \notin R_{\bar{q}}$, then $\bar{q}+e_{j} \notin \Omega$. It might appear that (3) is always true, but even though the transition from $\bar{q}$ to $\bar{q}+e_{j}$ is blocked by the policy, there could be some other path to get to the state $\bar{q}+e_{j}$. Therefore, (3) is a property of the policy.

If the condition in Remark 1 holds, the stationary state solution to the equation set (2) is (see e.g. [8, p. 160] or [9])

$$
\pi_{q}=\pi_{q_{1}, \ldots, q_{K}}=G^{-1} \prod_{j=1}^{K} \frac{1}{q_{j} !} \rho_{j}^{q_{j}} 1_{\bar{q} \in \Omega}
$$

where $G=\sum_{\bar{q} \in \Omega} \prod_{j=1}^{K} \frac{1}{q_{j} !} \rho_{j}^{q_{j}}, \rho_{j}=\frac{\lambda_{j}}{\mu_{j}}$.

Let us assume that blocking a call of type $j$ creates $\omega_{j}$ units of cost, and that calls are blocked if there are not enough capacity units. In the stationary state the process (2) generates the average cost $R$ where

$$
R=\frac{T}{N} g, g=\sum_{\bar{q} \in \Omega} r_{\bar{q}} \pi_{\bar{q}}, r_{\bar{q}}=\sum_{j \notin R_{\bar{q}}} \omega_{j} \lambda_{j}
$$

The number $g$ is the average cost rate and $r_{\bar{q}}$ is the average cost rate in the state $\bar{q}$.

\section{Charging by Shadow Prices in A Multiservice LOSS SYSTEM}

\section{A. Average costs}

The optimal pricing policy in congestion pricing is that the operator charges shadow prices - the expectations of future losses from blocked traffic. The shadow prices can be computed in the following way.

The multiservice loss system approaches a steady state and in the steady state it generates constant cost in each time step. The initial state determines how much cost the process generates before reaching the steady state and in most cases rather few steps from the beginning of the process determine quite well how much costs differ when the process is started in a given initial state. This cost difference can be calculated using Howard's equation (see [7])

$$
\mathbf{Q V}=g \mathbf{1}-\mathbf{r}
$$

where $\mathbf{Q}$ is the instantaneous transition matrix of a Markov chain, $\mathbf{V}$ is the vector of relative costs, $g$ is the average cost, and $\mathbf{r}$ is the vector of state cost rates. One method of solving Howard's equation is inverting $\mathbf{Q}$ :

$$
\mathbf{V}=\mathbf{Q}^{-\mathbf{1}}(g \mathbf{1}-\mathbf{r})
$$

Howard's equation for future relative cost expectations for a multi-service loss system in component form is:

$$
\begin{aligned}
& \sum_{j \in R_{\bar{q}}} \lambda_{j}\left(v\left(\bar{q}+e_{j}\right)-v(\bar{q})\right)-\sum_{j=1}^{K} \mu_{j} q_{j}\left(v(\bar{q})-v\left(\bar{q}-e_{j}\right)\right) \\
& =g-\sum_{j \notin R_{\bar{q}}} \lambda_{j} \omega_{j} .
\end{aligned}
$$

The variable $v(\bar{q})$ is called relative cost. The exact solution to Howard's equation is not unique: it is possible to add a constant to $v(\bar{q})$. The state-dependent link shadow price for traffic class $k$ is $p_{k}(\bar{q})=v_{\bar{q}+e_{j}}-v_{\bar{q}}$, see [7] formula (7).

If the dimension of the multiservice loss system is small, we can simply invert matrix $\mathbf{Q}$ and solve Howard's equation. If the dimension is very large this method becomes impractical and approximate methods are often proposed. One such method is in [7]. The authors select a small-dimensional basis, compute a projection to the basis, and obtain very good numerical results compared to earlier methods [10], [11], and [12]. We will here denote the capacity $d_{j}$ in Example 1 by $b_{j}$. In the special case $b_{j}=b, \mu_{j}=\mu$ and the policy of Example 1 the exact solution to Howard's equation is (see Appendix I):

$$
v(\bar{q})=\frac{g}{\mu \rho} \sum_{i=1}^{q} \sum_{m=0}^{q-i} \frac{(q-i) !}{(q-i-m) !} \rho^{-m}
$$

where $\rho=\sum_{k=1}^{K} \frac{\lambda_{k}}{\mu_{k}}$ and $q=\sum_{k=1}^{K} q_{k}$.

In more general cases we can start with an approximation and complete it into an exact solution in a way described in Appendix I. This way produces a series form solution.

In the case $b_{j}=b$ but $\mu_{j} \neq \mu_{k}$ for some $j$ and $k$, the following closed form approximation seems fairly good

$$
\tilde{v}(\bar{q})=\sum_{j=1}^{K} \frac{q_{j}}{q} \frac{g}{\mu_{j} \rho} \sum_{i=1}^{q} \sum_{m=0}^{q-i} \frac{(q-i) !}{(q-i-m) !} \rho^{-m} .
$$

In the general case where $b_{j} \neq b_{k}$ for some $j$ and $k$ and $\mu_{j} \neq \mu_{k}$ for some $j$ and $k$, a rather natural approximation to start from is

$\hat{v}(\bar{q})=\sum_{j=1}^{K} \frac{b_{j} q_{j}}{c}\left(\frac{b_{j}}{b}\right)^{2} \frac{g}{\mu_{j} \rho} \sum_{i=1}^{\left[\frac{c}{b_{j}}\right]} \sum_{m=0}^{\left[\frac{c}{b_{j}}\right]-i} \frac{\left(\left[\frac{c}{b_{j}}\right]-i\right) !}{\left(\left[\frac{c}{b_{j}}\right]-i-m\right) !} \rho_{j}^{-m}$ where $[x]$ means the largest integer smaller than $x$

$$
b=\sum_{j=1}^{K} b_{j}, \rho_{j}=\left(\frac{b}{b_{j}}\right)^{2} \rho
$$




$$
\rho=\sum_{k=1}^{K} \frac{\lambda_{k}}{\mu_{k}}\left(\frac{b_{k}}{b}\right)^{2} \text { and } c=\sum_{k=1}^{K} b_{k} q_{k} .
$$

These approximations are derived in Appendix I. They are simple to calculate and do not involve any matching of parameters. They are not expected to be as good as in [7] where a large number of free parameters are adjusted to specific numeric values of the loss system. If the shadow prices are used in pricing, we do not need high precision: the input parameters are not known precisely and the price will be rounded to a less precise figure.

In case better accuracy is needed we can calculate more terms in the following way. Any first order approximation $u(\bar{q})$ of $v(\bar{q})$ can be completed to an exact solution of (8) by the following procedure. Let us define recursively

$$
\begin{aligned}
& f_{1, j}(\bar{q})=1-\Delta_{j} u(\bar{q}) \text { and for } n \geq 1, \\
& f_{n+1, j}(\bar{q})=-\sum_{\substack{k=1 \\
k \neq j}}^{K} \Delta_{k} \frac{1}{\mu_{j}} h\left(f_{n, j}(\bar{q}), q_{j}, \rho_{j}\right)
\end{aligned}
$$

where $\rho_{j}=\lambda_{j} / \mu_{j}$ and

$$
\begin{aligned}
& h\left(f(\bar{q}), q_{j}, \rho\right)=\frac{1}{\rho} \sum_{i=1}^{q_{j}} \sum_{m=0}^{q_{j}-i} f\left(\bar{q}-(i+m) e_{j}\right) \frac{\left(q_{j}-i\right) !}{\left(q_{j}-i-m\right) !} \rho^{-m}, \\
& \Delta=\sum_{j=1}^{K} \Delta_{j}, \\
& \Delta_{j} f(\bar{q})=\lambda_{j}\left(f\left(\bar{q}+e_{j}\right)-f(\bar{q})\right)-\mu_{j} q_{j}\left(f(\bar{q})-f\left(\bar{q}-e_{j}\right)\right) .
\end{aligned}
$$

The function

$$
V_{\bar{q}}=g u(q)+g \sum_{j=1}^{K} \sum_{n=1}^{\infty} \frac{1}{\mu_{j}} h\left(f_{n, j}(\bar{q}), q_{j}, \rho_{j}\right)
$$

satisfies Howard's equation (8). An example of (14) is calculated in Appendix I starting from the approximation (10).

If the operator charges the user with the shadow prices and the network is in the steady state, the distribution of the cost to the user are calculated by multiplying the steady-state probabilities with the shadow prices.

\section{B. Cost Distribution using Shadow Prices}

The relative costs, and consequently the shadow prices, are transient phenomena. After a short time the system will be close to the steady state and producing the average cost rate in (5) regardless of what was the original state. More important parameter than the shadow price is the distribution of the cost in the future. It is not a transient phenomenon and it reflects the differences of using different prices on the services. As the average cost rate depends on the arrival rates $\lambda_{i}$ and the costs $\omega_{i}$, we may expect the cost distribution also to depend on these parameters. It is interesting to see if offering more services gives smaller variance in the cost distribution than offering fewer services. We can calculate the cost distribution by solving a set of recursion equations with the initial state of an empty system. If the system is not empty, there comes the transient distribution giving the average costs that are calculated with Howard's equation. These transient costs stay constant and become ignorable in the limit, thus starting from an empty system gives the same limit distribution.

In order to calculate the expected cost distribution up to a finite time horizon $T$ we describe the process using a state vector $(n, \bar{q}, r)$ and its probability $s_{n, \bar{q}, r}$. Then cost is treated as a state variable and cost distributions can be obtained.

Let $s_{n, \bar{q}, r}$ be the probability that the state is $\left(n, q_{1}, \ldots, q_{K}\right)$ and that the cost vector has the value $r$. Let $r$ be here a nonnegative integer and let us require that each $\omega_{j}$ is also an integer. Naturally we also require

$$
s_{n, q_{1}, \ldots, q_{K}}=\sum_{r=0}^{\infty} s_{n, q_{1}, \ldots, q_{K}, r} .
$$

We can derive a recursion equation set for the cost distribution by adding the parameter $r$ to (2):

$$
\begin{aligned}
& s_{n+1, q_{1}, \ldots, q_{K}, r}=s_{n, q_{1}, \ldots, q_{K}, r}-\sum_{j=1}^{K} \frac{\lambda_{j} T}{N} s_{n, q_{1}, \ldots, q_{K}, r} \\
& +\sum_{j=1}^{K} \frac{\lambda_{j} T}{N} s_{n, q_{1}, \ldots, q_{K}, r-\omega_{j}} 1_{j \notin R_{\bar{q}}} \\
& -\sum_{j=1}^{K} \frac{\mu_{j} T}{N} q_{j} s_{n, q_{1}, \ldots, q_{K}, r}+\sum_{j=1}^{K} \frac{\lambda_{j} T}{N} s_{n, q_{1}, \ldots, q_{j}-1, \ldots, q_{K}, r} 1_{j \in R_{\bar{q}-e_{j}}} \\
& \quad+\sum_{j=1}^{K} \frac{\mu_{j} T}{N}\left(q_{j}+1\right) s_{n, q_{1}, \ldots, q_{j}+1, \ldots, q_{K}, r}, \\
& s_{n, q_{1}, \ldots, q_{K}}=\sum_{r=0}^{\infty} s_{n, q_{1}, \ldots, q_{K}, r}, \sum_{\bar{q} \in \Omega} s_{n, q_{1}, \ldots, q_{K}}=1, \\
& 0 \leq s_{n, q_{1}, \ldots, q_{K}, r} \leq 1 .
\end{aligned}
$$

When (16) is summed over $r$, the process (2) is obtained.

The exact solution to (16) is calculated in Appendix II for the policy of Example 2.

Theorem 1. Assume that the policy is as in Example 2. Equation set (16) has a limit solution of the form

$$
s_{t, \bar{q}, r}=\sum_{\substack{r_{1}=\ldots=r_{K}=0 \\ r=\sum_{i=1}^{K} \omega_{i} r_{i}}}^{\infty} s_{t, r_{1}, \ldots, r_{K}} s_{t, \bar{q}, r_{1}, \ldots, r_{K}}
$$

where

$$
\begin{aligned}
& s_{t, \bar{q}, r_{1}, \ldots, r_{K}}=\sum_{i=1}^{K} s_{t, \bar{q}_{i}, r_{i}} G^{-1} \prod_{\substack{j=1 \\
j \neq i}}^{K} \frac{\rho_{j}^{q_{j}}}{q_{j} !} \\
& s_{t, r_{1}, \ldots, r_{K}}=A \prod_{i=1}^{K} \frac{1}{r_{i} !}\left(t \lambda_{i}\right)^{r_{i}} e^{-t \sum_{i=1}^{K} \lambda_{i}}
\end{aligned}
$$


$s_{t, q_{i}, r_{i}}=\frac{1}{q_{i} !} f_{i}\left(q_{i}, r_{i}, t\right) g_{i}\left(r_{i}, t\right)$,

$G^{-1}$ is from (4). The functions $f_{i}\left(q_{i}, r_{i}, t\right)$ and $g_{i}\left(r_{i}, t\right)$ are complicated and given in Appendix II.

For the policy in Example 1 we may use an approximation

$$
C_{i}=C_{i}(q)=\max _{q_{i}} q_{i} \leq \frac{C}{b_{i}}-\sum_{\substack{m=1 \\ m \neq i}}^{K} \frac{b_{m}}{b_{i}} q_{m}
$$

\section{in Theorem 1.}

The formula (20) describes how the cost distribution depends on the arrival rates $\lambda_{i}$ and service costs $\omega_{i}$. The solution (17) has an exact form given in Appendix II but it is very complicated. This raises the question whether a simpler pricing scheme could be used instead of shadow prices.

\section{A Simpler PRICING SCHEME}

As was seen in Section III, it is difficult to calculate the shadow prices from (8) and the cost distribution from (16). This section presents a simpler pricing scheme that overcomes these difficulties while producing the same average costs. system

A. Different cost distributions of a multiservice loss

One way is to use a product:

$$
\begin{aligned}
& s_{n, \bar{q}, r}=s_{n, q_{1}, \ldots, q_{K}, r}=s_{n, r}(\bar{q}) s_{n, q_{1}, \ldots, q_{K}} \\
& s_{n, r}(\bar{q})=0 \text { if } r<0,
\end{aligned}
$$

which satisfies the recursion equation

$$
\begin{aligned}
& s_{n+1, \bar{q}, r}=s_{n, \bar{q}, r}+s_{n, r}(\bar{q}) \Delta s_{n, \bar{q}}-\frac{T}{N} \sum_{j \notin R_{\bar{q}}} \lambda_{j} s_{n, \bar{q}, r}+\frac{T}{N} \sum_{j \notin R_{\bar{q}}} \lambda_{j} s_{n, \bar{q}, r-\omega_{j}} \\
& s_{n, q_{1}, \ldots, q_{K}}=\sum_{r=0}^{\infty} s_{n, q_{1}, \ldots, q_{K}, r}, \sum_{q \in \Omega} s_{n, q_{1}, \ldots, q_{K}}=1 \\
& 0 \leq s_{n, q_{1}, \ldots, q_{K}, r} \leq 1 .
\end{aligned}
$$

The operator $\Delta$ is shorthand to the infinitesimal transition matrix of a multiservice loss system:

$$
\begin{aligned}
& \Delta f_{n, q_{1}, \ldots, q_{K}}=-\sum_{j \in R_{q}} \frac{\lambda_{j} T}{N} f_{n, q_{1}, \ldots, q_{K}}-\sum_{j=1}^{K} \frac{\mu_{j} T}{N} q_{j} f_{n, q_{1}, \ldots, q_{K}} \\
& +\sum_{j \in R_{q-e_{j}}} \frac{\lambda_{j} T}{N} f_{n, q_{1}, \ldots, q_{j}-1, \ldots, q_{K}} \\
& +\sum_{j=1}^{K} \frac{\mu_{j} T}{N}\left(q_{j}+1\right) f_{n, q_{1}, \ldots, q_{j}+1, \ldots, q_{K}} .
\end{aligned}
$$

When (23) is summed over $r$, it gives (2). This is seen as follows:

$$
s_{n, q_{1}, \ldots, q_{K}}=\sum_{r=0}^{\infty} s_{n, q_{1}, \ldots, q_{K}, r}=s_{n, \bar{q}} \sum_{r=0}^{\infty} s_{n, r}(\bar{q}),
$$

thus $\sum_{r=0}^{\infty} s_{n, r}(\bar{q})=1$.

$$
\sum_{r=0}^{\infty} s_{n, r-\omega_{j}}(\bar{q})=\sum_{r^{\prime}=-\omega_{j}}^{\infty} s_{n, r^{\prime}}(\bar{q})=\sum_{r^{\prime}=0}^{\infty} s_{n, r^{\prime}}(\bar{q}),
$$

i.e., $\sum_{r=0}^{\infty}\left(-\sum_{j \notin R_{q}} \lambda_{j} s_{n, \bar{q}, r}+\sum_{j \notin R_{q}} \lambda_{j} s_{n, \bar{q}, r-\omega_{j}}\right)=0$ and we obtain

(2) in the form:

$$
s_{n+1, \bar{q}}=\sum_{r=0}^{\infty} s_{n+1, \bar{q}, r}=s_{n, \bar{q}}+\Delta s_{n, \bar{q}} .
$$

Another way is to take a slightly more general form:

$$
s_{n, \bar{q}, r}=a s_{n, r}(\bar{q}) s_{n, q_{1}, \ldots, q_{K}}+(1-a) v_{n, q_{1}, \ldots, q_{K}, r}
$$

where $a \in[0,1]$ and

$$
\begin{aligned}
& \lim _{n \rightarrow \infty}\left(v_{n+1, \bar{q}, r}-v_{n, \bar{q}, r}\right)=0, \sum_{r=0}^{\infty} v_{n, \bar{q}, r}=s_{n, \bar{q}}, \\
& \lim _{n \rightarrow \infty} \frac{v_{n, \bar{q}, r}}{s_{n, r}(\bar{q})}=0 .
\end{aligned}
$$

The corresponding recursion equation

$$
\begin{aligned}
& s_{n+1, \bar{q}, r}=s_{n, \bar{q}, r}+a s_{n, r}(\bar{q}) \Delta s_{n, \bar{q}}+(1-a) \Delta v_{n, \bar{q}, r} \\
& -\frac{T}{N} \sum_{j \notin R_{\bar{q}}} \lambda_{j} s_{n, \bar{q}, r}+\frac{T}{N} \sum_{j \notin R_{\bar{q}}} \lambda_{j} s_{n, \bar{q}, r-\omega_{j}} \\
& s_{n, q_{1}, \ldots, q_{K}}=\sum_{r=0}^{\infty} s_{n, q_{1}, \ldots, q_{K}, r}, \sum_{q \in \Omega} s_{n, q_{1}, \ldots, q_{K}}=1, \\
& 0 \leq s_{n, q_{1}, \ldots, q_{K}, r} \leq 1 .
\end{aligned}
$$

also gives (2) when summed over $r$. To see this, let us notice that the last two terms of (28) cancel when summed over $r$ and that

$$
\sum_{r=0}^{\infty} \Delta v_{n, \bar{q}, r}=\Delta \sum_{r=0}^{\infty} v_{n, \bar{q}, r}=\Delta s_{n, \bar{q}}=0 .
$$

Then $a \sum_{r=0}^{\infty} s_{n, r}(\bar{q})=1$ because the term

$$
\sum_{r=0}^{\infty}(1-a) v_{n, \bar{q}, r} \text { disappears in the limit } n \rightarrow \infty \text {. }
$$

Lemma 1. The set of equations (16), (23) and (28) all generate the cost rate $R$ in (5).

The proposed simpler pricing scheme is pricing the connections with the future expectations of the process (23) instead of by the future expectations of the process (16). In 
shadow prices (16) all states $\bar{q}$ are associated with a positive shadow price because there is some future expectation that the system starting from $\bar{q}$ will be blocking traffic in the future, and consequently future expectations of costs are positive. In (23) only such states that block give any charge. We will show this after Theorems 2 and 3 below. While shadow prices are optimal in a certain sense, the differences between shadow prices and pricing by future expectations of (23) are small. User's bills are in any case rounded, user traffic is measured, network congestion state is estimated and in reality shadow prices cannot be very exact.

\section{B. Cost distribution for the simpler pricing scheme}

The recursion equations (23) can be solved exactly because they are created to satisfy the product form solution (22). The limit distribution to the recursion equations (28) can be solved similarly. The part $v_{n, \bar{q}, r}$ disappears in the limit.

Theorem 2. Assume that the process (23) satisfies $s_{0, \bar{q}, r}=0$ if $r \neq 0$. The solution to (23) has the form

$$
S_{n, q_{1}, \ldots, q_{K}, r}=\left(\sum_{\substack{r_{1}=\ldots=r_{K}=0 \\\left(r_{1}, \ldots, r_{K}\right) \in A_{r, \bar{q}}}}^{n} C_{n, \ldots q_{K}, r}\right) \pi_{n, \bar{q}}
$$

where

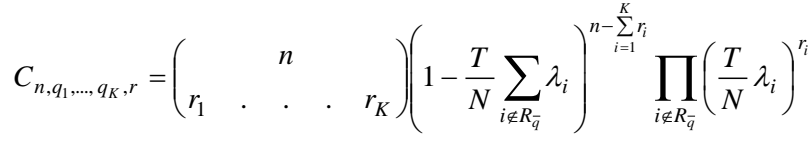

$$
\begin{aligned}
& A_{r, \bar{q}}=\left\{\left(r_{1}, \ldots, r_{K}\right) \mid \sum_{i=1}^{K} \omega_{i} r_{i}=r, \quad r_{i}=0 \quad \text { if } \quad i \in R_{\bar{q}}\right\}
\end{aligned}
$$

and $\pi_{n, \bar{q}} \rightarrow \pi_{\bar{q}}$ when $n \rightarrow \infty, \pi_{\bar{q}}$ is given by (4).

It is also possible to derive the continuous limit:

Theorem 3. The continuous time limit function for (30) when $N \rightarrow \infty, n=x N, t=x T$ is

$$
s_{t, q_{1}, \ldots, q_{K}, r}=\left(\sum_{\substack{r_{1}=\ldots=r_{K}=0 \\\left(r_{1}, \ldots, r_{K}\right) \in A_{r, \bar{q}}}}^{\infty} \prod_{i \notin R_{\bar{q}}} \frac{1}{r_{i} !}\left(t \lambda_{i}\right)^{r_{i}}\right) e^{-t \sum_{i \notin R_{\bar{q}}} \lambda_{i}} \pi_{\bar{q}} .
$$

Let us note that (30) is not a solution to (16). The formula (30) has the form of a product

$$
s_{n, q_{1}, \ldots ., q_{K}, r}=s_{n, r}(\bar{q}) \pi_{q} .
$$

Then the detailed balance conditions for each $j$ and $r$ in the limit $n \rightarrow \infty$

$$
\lim _{n \rightarrow \infty} \mu_{i}\left(q_{i}+1\right) s_{n, q_{1}, \ldots, q_{j}+1, \ldots, q_{K}, r}=\lim _{n \rightarrow \infty} \lambda_{i} s_{n, q_{1}, \ldots, q_{K}, r}
$$

and

$$
\lim _{n \rightarrow \infty} \mu_{i} q_{i} s_{n, q_{1}, \ldots, q_{K}, r}=\lim _{n \rightarrow \infty} \lambda_{i} s_{n, q_{1}, \ldots, q_{i}-1, \ldots, q_{K}, r}
$$

do not hold in (16), since $\pi_{q}$ satisfies them, but if for some $q$ holds $R_{q} \neq R_{q+e_{i}}$, then $s_{n, r}\left(q+e_{i}\right) \neq s_{n, r}(q)$.

Let us check that (31) produces the correct cost.

Lemma 2. The solution (31) satisfies

$$
\sum_{r=0}^{\infty} r s_{t, \bar{q}, r}=t r_{\bar{q}} \pi_{\bar{q}} \text {. }
$$

Remark 2. Let us calculate directly two easy examples:

a) If $R_{q}=\{1,2\}$ and $\omega_{1}=\omega_{2}=1$, then

$$
\begin{aligned}
& \sum_{r=0}^{\infty} r s_{t, q_{1}, \ldots, q_{K}, r} \\
&= \sum_{r=0}^{\infty} r \sum_{r_{1}=0}^{r} \frac{1}{r_{1} !} \frac{1}{\left(r-r_{1}\right) !}\left(x T \lambda_{1}\right)^{r_{1}}\left(x T \lambda_{2}\right)^{r-r_{1}} e^{-x T\left(\lambda_{1}+\lambda_{2}\right)} \pi_{\bar{q}} \\
&=\sum_{r=0}^{\infty} \frac{1}{(r-1) !}(x T)^{r}\left(\lambda_{1}+\lambda_{2}\right)^{r} e^{-x T\left(\lambda_{1}+\lambda_{2}\right)} \pi_{\bar{q}} \\
&=x T\left(\lambda_{1}+\lambda_{2}\right) \pi_{\bar{q}}=t r_{\bar{q}} \pi_{\bar{q}} .
\end{aligned}
$$

b) If $R_{q}=\{1\}$, then the multinomial recurrence formula

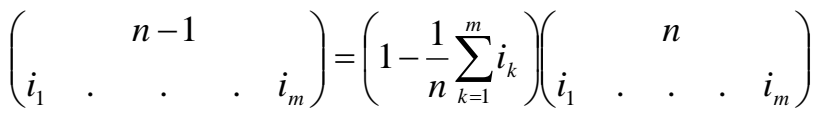

for the binomial term gives for $n>1$ :

$$
\begin{aligned}
& \sum_{r=0}^{\infty} r s_{n, q_{1}, \ldots, q_{K}, r}=\sum_{i_{i}=0}^{n} \omega_{i} i_{i}\left(\begin{array}{l}
n \\
i_{i}
\end{array}\right)\left(1-\frac{T}{N} \lambda_{1}\right)^{n-i_{1}}\left(\frac{T}{N} \lambda_{1}\right)^{i_{1}} \pi_{\bar{q}} \\
= & n \frac{T}{N} \omega_{1} \lambda_{1} \pi_{\bar{q}}
\end{aligned}
$$

Let us verfy that in Theorems 2 and 3 only such states that block give any charge: If the system is in a state that does not block, there is only the point $\left(r_{1}, \ldots, r_{K}\right)=(0, \ldots, 0)$ in $A_{r, \bar{q}}$. Thus, $s_{n, q, r}=0$ if $r \neq 0$.

Let us notice that the part corresponding to the arrival rates $\lambda_{i}$ and service costs $\omega_{i}$ is similar in (17) and (31), only in (17) there is the scaling factor $A$.

\section{CONCLUSIONS}

The user and the operator are interested in the variability of the cost of a connection caused by congestion pricing. The distribution of the cost of a connection to a user can be calculated in the ways given in Section III. 
An operator should know the cost distribution because it may have to add a price component to account for the risk of the actual costs exceeding the average costs. An exact solution for the distribution of costs to an operator over a longer time period can be obtained for a particular way of distributing costs to users, described by the recursion equation set (23). It is a slightly different pricing strategy than using shadow prices, but it gives the same incentives for users to lower their traffic demands. For shadow prices an exact expression could only be obtained for the simple blocking policy in Example 2. However, the structure of the cost distribution is very similar in (17) and (32), and (32) can be used as an approximation.

The pricing scheme in Theorems 2 and 3 does not much differ from the shadow pricing scheme. Pricing schemes that approximate shadow prices but are more simple have earlier been suggested by other authors, for instance [6] proposes a static pricing scheme. Exact shadow prices are found too complicated and simpler approximations are acceptable substitutes.

Theorems 1, 2 and 3 use the product form solution (4), which gives an exact solution to blocking probabilities for a certain class of routing policies in a circuit-switched network. The normalization constant $G$ is difficult to calculate but effective approximations do exist, or instance [14] and [15]. The expressions in Theorems 2 and 3 are rather similar to that of $G$ and similar effective calculation methods apply. As the cost distribution is only used for risk estimation and not for satisfying Quality of Service requirements, less precise methods are sufficient. Calculation of $G$ precisely is a NPcomplete problem and similar complexity is expected to the $r$ dependent part in (17) and (31), but as only the general shape of the distribution is needed, Monte Carlo simulation can give sufficient approximations for practical purposes in a reasonable time.

Theorems 1, 2 and 3 deal with the risk the operator is taking with congestion pricing. There is a general feeling that some price component to cover the risks is needed, but the case of risk in congestion pricing has not been much analyzed in the literature. The general aspect of risk is much wider than is treated in this paper. Many issues, such as growing demand and various aspects of uncertainty [16], [17] and [18] must be omitted in this paper.

\section{APPENDIX I: PROOFS OF SECTION II $A$.}

Proof of (9): Let $v(\bar{q})$ be as in (9), then

$$
v(\bar{q})-v\left(\bar{q}-e_{j}\right)=\frac{g}{\mu \rho} \sum_{m=0}^{q-1} \frac{(q-1) !}{(q-1-m) !} \rho^{-m} .
$$

In the policy of Example 1 holds $1_{c<C}=1_{j \in R_{\bar{q}}}$, thus

$$
\begin{aligned}
& \sum_{j=1}^{K} 1_{c<C} \lambda_{j}\left(v\left(\bar{q}+e_{j}\right)-v(\bar{q})\right)-\sum_{j=1}^{K} \mu q_{j}\left(v(\bar{q})-v\left(\bar{q}-e_{j}\right)\right) \\
& =\frac{g}{\mu \rho} \sum_{i=1}^{K} 1_{c<C} \lambda_{i} \sum_{m=0}^{q} \frac{q !}{(q-m) !} \rho^{-m}-\sum_{i=1}^{K} q_{i} \frac{g}{\rho} \rho \sum_{m=1}^{q} \frac{(q-1) !}{(q-m) !} \rho^{-m}
\end{aligned}
$$

$$
=\left\{\begin{array}{lll}
g & \text { if } & c<C \\
A & \text { if } & c=C
\end{array}\right.
$$

where

$$
A=-g \sum_{m=1}^{C / b} \frac{(C / b) !}{(C / b-m) !} \rho^{-m} .
$$

There is only one nonzero value for $\sum_{j \notin R_{\bar{q}}} \lambda_{j} \omega_{j}$ in this case and the value of $g$ in (5) sets $A$ to the value satisfying (8):

$$
A=g-\sum_{j \notin R_{\bar{q}}} \lambda_{j} \omega_{j} \cdot \square
$$

Motivation of (10) and (11): We show why the heuristic approximations (10) and (11) are natural and can give rather good results. Let us assume that at the axis $\bar{q}=q e_{j}$ the shadow price has the approximation

$p_{k}(\bar{q})=v\left(\bar{q}+e_{k}\right)-v(\bar{q}) \approx a_{k} \frac{g}{\mu \rho} \sum_{m=0}^{q_{j}} \frac{q_{j} !}{\left(q_{j}-m\right) !} \rho^{-m}$.

This is a generalization of the relative cost given by (9) and $a_{k}=1$ if every $\mu_{j}=\mu$. In the area when every call is accepted the recursion (8) gives for $\bar{q}=q e_{j}$

$$
\sum_{k=1}^{K} \lambda_{k} p_{k}(\bar{q})-\mu_{j} q_{j} p_{j}\left(\bar{q}-e_{j}\right)=g
$$

For the approximation (11) we get

$$
\begin{aligned}
& \sum_{k=1}^{K} \lambda_{k} a_{k} \frac{g}{\mu \rho} \sum_{m=0}^{q_{j}} \frac{q_{j} !}{\left(q_{j}-m\right) !} \rho^{-m}-\mu_{j} q_{j} a_{j} \frac{g}{\mu \rho} \sum_{m=0}^{q_{j}-1} \frac{\left(q_{j}-1\right) !}{\left(q_{j}-1-m\right) !} \rho^{-m} \\
& =\sum_{k=1}^{K} \lambda_{k} a_{k} \frac{g}{\mu \rho}+\left(\sum_{k=1}^{K} \lambda_{k} a_{k} \frac{g}{\mu \rho}-\mu_{j} a_{j} \frac{g}{\mu}\right) \sum_{m=0}^{q_{j}} \frac{q_{j} !}{\left(q_{j}-m\right) !} \rho^{-m}=g .
\end{aligned}
$$

This is satisfied if

$$
\begin{gathered}
\rho=\sum_{k=1}^{K} \lambda_{k} \frac{a_{k}}{\mu} \quad \text { and } 1=\mu_{j} a_{j} \frac{1}{\mu}, \\
\text { i.e., } a_{j}=\frac{\mu}{\mu_{j}}, \rho=\sum_{k=1}^{K} \frac{\lambda_{k}}{\mu_{k}} \text { where } \mu=\sum_{j=1}^{K} \mu_{j} .
\end{gathered}
$$

Proof of (14):

The following lemma holds.

Lemma 3:

$$
\Delta_{j} \frac{1}{\mu_{j}} h\left(f(\bar{q}), q_{j}, \rho_{j}\right)=f(\bar{q}) \text { for } \rho_{j}=\lambda_{j} / \mu_{j} .
$$

Proof:

$$
\begin{aligned}
& \rho_{j}\left(h\left(f\left(\bar{q}+e_{j}\right), q_{j}+1, \rho_{j}\right)-h\left(f(\bar{q}), q_{j}, \rho_{j}\right)\right) \\
& -q_{j}\left(h\left(f(\bar{q}), q_{j}, \rho_{j}\right)-h\left(f\left(\bar{q}-e_{j}\right), q_{j}-1, \rho_{j}\right)\right)
\end{aligned}
$$


$=\sum_{i=0}^{q_{j}} \sum_{m=0}^{q_{j}-i} f\left(\bar{q}-(i+m) e_{j}\right) \frac{\left(q_{j}-i\right) !}{\left(q_{j}-i-m\right) !} \rho_{j}^{-m}$

$-\sum_{i=1}^{q_{j}} \sum_{m=0}^{q_{j}-i} f\left(\bar{q}-(i+m) e_{j}\right) \frac{\left(q_{j}-i\right) !}{\left(q_{j}-i-m\right) !} \rho_{j}^{-m}$

$-q_{j} \frac{1}{\rho_{j}}\left(\sum_{i=1}^{q_{j}} \sum_{m=0}^{q_{j}-i} f\left(\bar{q}-(i+m) e_{j}\right) \frac{\left(q_{j}-i\right) !}{\left(q_{j}-i-m\right) !} \rho_{j}^{-m}\right)$

$+q_{j} \frac{1}{\rho_{j}}\left(\sum_{i=2}^{q_{j}} \sum_{m=0}^{q_{j}-i} f\left(\bar{q}-(i+m) e_{j}\right) \frac{\left(q_{j}-i\right) !}{\left(q_{j}-i-m\right) !} \rho_{j}^{-m}\right)$

$=\sum_{m=0}^{q_{j}} f\left(\bar{q}-m e_{j}\right) \frac{\left(q_{j}\right) !}{\left(q_{j}-m\right) !} \rho_{j}^{-m}$

$-q_{j} \frac{1}{\rho_{j}} \sum_{m=0}^{q_{j}-1} f\left(\bar{q}-(m+1) e_{j}\right) \frac{\left(q_{j}-1\right) !}{\left(q_{j}-1-m\right) !} \rho_{j}^{-m}$

$=\sum_{m=0}^{q_{j}} f\left(\bar{q}-m e_{j}\right) \frac{\left(q_{j}\right) !}{\left(q_{j}-m\right) !} \rho_{j}^{-m}$

$-\sum_{m=1}^{q_{j}} f\left(\bar{q}-m e_{j}\right) \frac{\left(q_{j}\right) !}{\left(q_{j}-m\right) !} \rho_{j}^{-m}=f(\bar{q})$.

By Lemma 3, $V_{\bar{q}}$ satisfies

$$
\begin{aligned}
& \Delta V_{\bar{q}}=g \sum_{j=1}^{K} \Delta_{j} u(q)+g \sum_{j=1}^{K} \sum_{n=1}^{\infty} \sum_{k=1}^{K} \Delta_{k} \frac{1}{\mu_{j}} h\left(f_{n, j}(\bar{q}), q_{j}, \rho_{j}\right) \\
& =g \sum_{j=1}^{K}\left(1-f_{1, j}(\bar{q})\right)+g \sum_{j=1}^{K} \sum_{n=1}^{\infty}\left(f_{n, j}(\bar{q})-f_{n+1, j}(\bar{q})\right) \\
& =g \sum_{j=1}^{K}\left(1-f_{1, j}(\bar{q})\right)+g \sum_{j=1}^{K} f_{1, j}(\bar{q})=g
\end{aligned}
$$

The value of $v(\bar{q})$ at the points when some class is blocked is already determined by the values of $v(\bar{q})$ at the points when no class is blocked. This means that we do not need to modify $V_{\bar{q}}$ so that it satisfies (8). Changing the operator $\Delta$ to

$$
\Delta=\sum_{j=1}^{K} \Delta_{j}
$$

$$
\Delta_{j} f(\bar{q})=1_{j \in R_{\bar{q}}} \lambda_{j}\left(f\left(\bar{q}+e_{j}\right)-f(\bar{q})\right)-\mu_{j} q_{j}\left(f(\bar{q})-f\left(\bar{q}-e_{j}\right)\right.
$$

the solution $V_{\bar{q}}$ necessarily satisfies (8):

$$
\Delta V_{\bar{q}}=g-\sum_{j \notin R_{\bar{q}}} \lambda_{j} \omega_{j} .
$$

This is so because before states block, the recursion equation is the same as in (13) and therefore the solution agrees with $V_{\bar{q}}$ for those values. At the border when states block, $V_{\bar{q}}$ is already determined by the values at unblocking states. Therefore Howard's equation must be satisfied at the blocking states.
As an example of (14) let us complete the approximation $\tilde{v}(\bar{q})$ in (10) to an exact solution of (8). Let us select

$$
u(\bar{q})=\frac{1}{\mu} h(1, q, \rho)
$$

where $q=\sum_{j=1}^{K} q_{j}, \mu=\sum_{j=1}^{K} \mu_{j}, \rho=\sum_{j=1}^{K} \frac{\lambda_{j}}{\mu_{j}}$ and

$h(f(q), q, \rho)=\frac{1}{\rho} \sum_{i=1}^{q} \sum_{m=0}^{q-i} f(q-i-m) \frac{(q-i) !}{(q-i-m) !} \rho^{-m}$.

Let $\Delta$ be as in (46). A similar calculation as in the proof of Lemma 3 shows that

$$
\begin{aligned}
& \Delta \frac{1}{\mu} h(1, q, \rho)=\sum_{j=1}^{K} \frac{\lambda_{j}}{\mu} \frac{1}{\rho} \sum_{m=0}^{q} \frac{q !}{(q-m) !} \rho^{-m} \\
& -\sum_{j=1}^{K} \frac{\mu_{j}}{\mu} q_{j} \sum_{m=1}^{q} \frac{(q-1) !}{(q-m) !} \rho^{-m} .
\end{aligned}
$$

Then

$$
\begin{aligned}
& f_{1, j}(\bar{q})=\left(\frac{\lambda_{j}}{\mu_{j}}-\frac{\lambda_{j}}{\mu}\right) \frac{1}{\rho} \sum_{m=0}^{q} \frac{q !}{(q-m) !} \rho^{-m} \\
&-\left(1-\frac{\mu_{j}}{\mu}\right) q_{j} \sum_{m=1}^{q} \frac{(q-1) !}{(q-m) !} \rho^{-m} .
\end{aligned}
$$

At any coordinate axis $\bar{q}=q e_{j}$ holds $\tilde{v}(q)=g u(\bar{q})$. The complete solution to (8) is

$$
V_{\bar{q}}=g \frac{1}{\mu} h(1, q, \rho)+g \sum_{j=1}^{K} \sum_{n=1}^{\infty} \frac{1}{\mu_{j}} h\left(f_{n, j}(\bar{q}), q_{j}, \rho_{j}\right) \text {. }
$$

\section{APPENDIX II: PROOF OF THEOREM 1}

For notational simplicity, we write $\bar{q}$ simply as $q$. We only intend to prove that (16) has a solution of the form (17) and do this by constructing such a solution. The solution turns out to be very complicated and not of practical usage. However, Lemma 4 below is interesting in its own right. The equation is reoccurring often in Markov chains with a cost parameter and the solution is not that easy to derive.

Lemma 4. Let us assume that $f: \mathbf{N} \rightarrow \mathbf{R}$ satisfies the recursion equation

$$
\begin{aligned}
& f(q+2)-(q+a) f(q+1)+\rho(q+1) f(q)=0, \\
& f(q)=0 \text { if } q<0,
\end{aligned}
$$

where $q \in \mathbf{Z}, \rho, a \in \mathbf{R}, a-\rho \notin \mathbf{N}$ and $\rho \neq 0$.

Then the function $f$ is

$$
\begin{aligned}
f(q)= & \sum_{j=1}^{q+\lfloor a-\rho\rfloor} \gamma_{j} \Gamma(q+a-\rho-j) \\
& +C \sum_{j=1}^{q+\lceil a-\rho\rceil} \gamma_{j} \Gamma(q+a-\rho-j)
\end{aligned}
$$


where

$$
\begin{aligned}
& \alpha_{1}=\gamma_{1} \rho(\rho+2-a), \alpha_{i+1}=\frac{\rho}{i} \alpha_{i}(2+\rho-a+i), \\
& i \geq 1, \text { and } \gamma_{i+1}=\frac{\alpha_{i}}{i}, i \geq 1 \text { and } C \text { is a real number. }
\end{aligned}
$$

Proof: Let us define

$$
\begin{gathered}
f_{i}(q)=\sum_{j=1}^{i} \gamma_{j} \Gamma(q+a-\rho-j) \\
\text { where } \Gamma(x+1)=\prod_{j=1}^{\lfloor x\rfloor}(j+x-\lfloor x\rfloor) .
\end{gathered}
$$

Write

Then

$$
A_{i}=f_{i}(q+2)-(q+a) f_{i}(q+1)+\rho(q+1) f_{i}(q) .
$$

$$
\begin{aligned}
& A_{i+1}-A_{i}=\gamma_{i+1} \Gamma(q+2+a-\rho-i-1) \\
& -(q+a) \gamma_{i+1} \Gamma(q+1+a-\rho-i-1) \\
& +\rho(q+1) \gamma_{i+1} \Gamma(q+a-\rho-i-1) .
\end{aligned}
$$

From $f_{1}(q)=\gamma_{1} \Gamma(q+a-\rho-1)$ we get the initial step to the induction:

$$
A_{1}=\gamma_{1} \rho(\rho+2-a) f_{1}(q)=\alpha_{1} \Gamma(q+a-\rho-1)
$$

when we define $\alpha_{1}=\gamma_{1} \rho(\rho+2-a)$. Let the induction assumption be that for $j=1, \ldots, i$ and for some numbers $\alpha_{j}$ holds

$$
A_{j}=\alpha_{j} \Gamma(q+a-\rho-j)
$$

Then for $j=i+1$ we get

$$
\begin{aligned}
& A_{i+1}-A_{i} \\
& =\gamma_{i+1} \Gamma(q+a-\rho-i-1)((q+a-\rho-i)(q+a-\rho-i-1) \\
& \quad-(q+a)(q+a-\rho-i-1)+\rho q+\rho) \\
& =\gamma_{i+1} \Gamma(q+a-\rho-i-1)(-i q-(a-\rho-i-1)(\rho+i)+\rho) .
\end{aligned}
$$

By the induction assumption

$$
A_{i}=\alpha_{i}(q+a-\rho-i-1) \Gamma(q+a-\rho-i-1) .
$$

Then

$$
\begin{aligned}
& A_{i+1}=\Gamma(q+a-\rho-i-1)\left(\left(\alpha_{i}-\gamma_{i+1} i\right) q\right. \\
& \left.+\alpha_{i}(a-\rho-i-1)-\gamma_{i+1}(a-\rho-i-1)(\rho+i)+\gamma_{i+1} \rho\right) \\
& =\alpha_{i+1} \Gamma(q+a-\rho-i-1)
\end{aligned}
$$

when we choose

$$
\gamma_{i+1}=\frac{\alpha_{i}}{i} \text { and } \alpha_{i+1}=\frac{\rho}{i} \alpha_{i}(2+\rho-a+i) .
$$

By induction we can continue so long that $q$ is removed from the Gamma function and we have a scalar. The scalar is unfortunately not usually the correct initial value. Let us define

$$
\begin{aligned}
& A_{\lfloor a-\rho\rfloor+q}=\alpha_{\lfloor a-\rho\rfloor+q} \Gamma(a-\rho-\lfloor a-\rho\rfloor) \\
& A_{\lceil a-\rho\rceil+q}=\alpha_{[a-\rho]+q} \Gamma(a-\rho-[a-\rho]) .
\end{aligned}
$$

These are scalars, i.e., not functions of $q$. If $a-\rho \notin \mathbf{N}$, we get two different scalars that can be weighted to that their linear combination is the desired initial value (zero). Thus

$f(q)=\sum_{j=1}^{q+\lfloor a-\rho\rfloor} \gamma_{j} \Gamma(q+a-\rho-j)+C \sum_{j=1}^{q+\lceil a-\rho\rceil} \gamma_{j} \Gamma(q+a-\rho-j)$

satisfies (51) if $C=-A_{\lfloor q+a-\rho\rfloor} / A_{\lceil q+a-\rho\rceil}$. The parameter $\gamma_{1}$ is a free parameter and we can set $\gamma_{1}=1$.

Proof of Theorem 1: Let us use the trial

$$
s_{n, q, r}=\sum_{\substack{r_{1}=\ldots=r_{K}=0 \\ r=\sum_{i=1}^{K} \omega_{i} r_{i}}}^{\infty} s_{n, q, r_{1}, \ldots, r_{K}}
$$

and require that each term of the sum satisfies (16). Using the property (3), we can change the position of $1_{j \in R_{\bar{q}}}$ and rewrite (16) in the following form:

$$
\begin{aligned}
& s_{n+1, q, r_{1}, \ldots, r_{K}}=s_{n, q, r_{1}, \ldots, r_{K}} \\
& -\frac{T}{N} \sum_{i=1}^{K} \mu_{i} q_{i} s_{n, q, r_{1}, \ldots, r_{K}}-\frac{T}{N} \sum_{i=1}^{K} \lambda_{i} s_{n, q, r_{1}, \ldots, r_{K}} \\
& +\frac{T}{N} \sum_{i=1}^{K} \mu_{i}\left(q_{i}+1\right) s_{n, q+e_{i}, r_{1}, \ldots, r_{K}} 1_{i \in R_{q}} \\
& +\frac{T}{N} \sum_{i=1}^{K} \lambda_{i} s_{n, q-e_{i}, r_{1}, \ldots, r_{K}}+\frac{T}{N} \sum_{i=1}^{K} \lambda_{i} s_{n, q, r_{1}, \ldots, r_{i}-1, \ldots, r_{K}} 1_{i \notin R_{q}} .
\end{aligned}
$$

Let us look for a solution of the type

$$
s_{n, r_{1}, \ldots, r_{K}} s_{t, q, r_{1}, \ldots, r_{K}}=s_{n, r_{1}, \ldots, r_{K}} \sum_{i=1}^{K} s_{t, q_{i}, r_{i}} G^{-1} \prod_{\substack{j=1 \\ j \neq i}}^{K} \frac{\rho_{j}^{q_{j}}}{q_{j} !}
$$

where

$$
\begin{aligned}
& s_{n+1, r_{1}, \ldots, r_{K}}=s_{n, r_{1}, \ldots, r_{K}}-\frac{T}{N} \sum_{i=1}^{K} a_{i} \lambda_{i} s_{n, r_{1}, \ldots, r_{K}}+\frac{T}{N} \sum_{i=1}^{K} a_{i} \lambda_{i} s_{n, r_{1}, \ldots, r_{i}-1, \ldots, r_{K}} \\
& s_{0, r_{1}, \ldots, r_{K}}=\delta_{r_{1}=\ldots=r_{K}=0} .
\end{aligned}
$$

Summing over $r_{i}$ gives

$s_{n, r}=\left(\sum_{\substack{r_{1}=\ldots=r_{K}=0 \\ r=\sum_{i=1}^{K} \omega_{i} r_{i}}}^{\infty}\left(\begin{array}{cccc} & & n & \\ r_{1} & . & r_{K}\end{array}\right)\left(1-\frac{T}{N} \sum_{i=1}^{K} a_{i} \lambda_{i}\right)^{n-\sum_{i=1}^{K} r_{i}} \prod_{i=1}^{K}\left(\frac{T}{N} a_{i} \lambda_{i}\right)^{r_{i}}\right)$. 
Taking the continuous limit of this expression gives (20). Let us modify (62) by adding and removing terms:

$$
\begin{aligned}
& s_{n+1, r_{1}, \ldots, r_{K}} s_{q, r_{1}, \ldots, r_{K}}=s_{n, r_{1}, \ldots, r_{K}} s_{q, r_{1}, \ldots, r_{K}} \\
& -\frac{T}{N} \sum_{i=1}^{K} a_{i} \lambda_{i} s_{n, r_{1}, \ldots, r_{K}} s_{q, r_{1}, \ldots, r_{K}}+\frac{T}{N} \sum_{i=1}^{K} a_{i} \lambda_{i} s_{n, r_{1}, \ldots, r_{i}-1, \ldots, r_{K}} s_{q, r_{1}, \ldots, r_{K}} \\
& +\frac{T}{N} \sum_{i=1}^{K} a_{i} \lambda_{i} s_{n, r_{1}, \ldots, r_{K}} s_{q, r_{1}, \ldots, r_{K}}-\frac{T}{N} \sum_{i=1}^{K} a_{i} \lambda_{i} s_{n, r_{1}, \ldots, r_{i}-1, \ldots, r_{K}} s_{q, r_{1}, \ldots, r_{K}} \\
& -\frac{T}{N} \sum_{i=1}^{K} \mu_{i} q_{i} s_{n, q, r_{1}, \ldots, r_{K}}-\frac{T}{N} \sum_{i=1}^{K} \lambda_{i} s_{n, q, r_{1}, \ldots, r_{K}} \\
& +\frac{T}{N} \sum_{i=1}^{K} \mu_{i}\left(q_{i}+1\right) s_{n, q+e_{i}, r_{1}, \ldots, r_{K}} 1_{i \in R_{q}}+\frac{T}{N} \sum_{i=1}^{K} \lambda_{i} s_{n, q-e_{i}, r_{1}, \ldots, r_{K}} \\
& \quad+\frac{T}{N} \sum_{i=1}^{K} \lambda_{i} s_{n, q, r_{1}, \ldots, r_{i}-1, \ldots, r_{K}} 1_{i \notin R_{q}} .
\end{aligned}
$$

The first four terms give zero because of (64), thus the other terms also sum to zero. Let us take the continuous limit $n / N \rightarrow t / T$ and define

$$
h_{i}\left(r_{i}, t\right)=\lim _{n \rightarrow t} \frac{s_{n, r_{1}, \ldots, r_{i}-1, \ldots, r_{K}}}{s_{n, r_{1}, \ldots, r_{K}}}=\frac{r_{i}}{t a_{i} \lambda_{i}},
$$

where $t$ is the continuous time parameter. Dividing (65) by $s_{n, r_{1}, \ldots, r_{K}}$ and inserting $h_{i}\left(r_{i}, t\right)$ and

$$
s_{t, q_{i}, r_{i}}=\frac{1}{q_{i} !} f_{i}\left(q_{i}, r_{i}, t\right) g_{i}\left(r_{i}, t\right)
$$

gives

$$
0=\sum_{i=1}^{K} \prod_{\substack{j=1 \\ j \neq i}}^{K} \frac{\rho_{j}^{q_{j}}}{q_{j} !} \lambda_{i} \theta_{i}
$$

where

$$
\begin{aligned}
\theta_{i}= & \left(a_{i}-1-a_{i} h_{i}\left(r_{i}, t\right)\right) \frac{1}{q_{i} !} f_{i}\left(q_{i}, r_{i}, t\right) g_{i}\left(r_{i}, t\right) \\
& -\rho_{i}^{-1} \frac{1}{\left(q_{i}-1\right) !} f_{i}\left(q_{i}, r_{i}, t\right) g_{i}\left(r_{i}, t\right) \\
& +\rho_{i}^{-1} \frac{1}{q_{i} !} f_{i}\left(q_{i}+1, r_{i}, t\right) g_{i}\left(r_{i}, t\right) 1_{i \in R_{q}} \\
& +\frac{1}{\left(q_{i}-1\right) !} f_{i}\left(q_{i}-1, r_{i}, t\right) g_{i}\left(r_{i}, t\right) \\
& +h_{i}\left(r_{i}, t\right) \frac{1}{q_{i} !} f_{i}\left(q_{i}, r_{i}-1, t\right) g_{i}\left(r_{i}-1, t\right) 1_{i \notin R_{q}} .
\end{aligned}
$$

Let us satisfy (68) by setting the $\theta_{i}$ to zero for every $i$, thus

$$
\begin{aligned}
& 0=f_{i}\left(q_{i}+1, r_{i}, t\right) g_{i}\left(r_{i}, t\right) 1_{i \in R_{q}} \\
& -\left(q_{i}+\rho_{i}\left(1-a_{i}+a_{i} h_{i}\left(r_{i}, t\right)\right)\right) f_{i}\left(q_{i}, r_{i}, t\right) g_{i}\left(r_{i}, t\right) \\
& +\rho_{i} q_{i} f_{i}\left(q_{i}-1, r_{i}, t\right) g_{i}\left(r_{i}, t\right)
\end{aligned}
$$

$$
+\rho_{i} h_{i}\left(r_{i}, t\right) f_{i}\left(q_{i}, r_{i}-1, t\right) g_{i}\left(r_{i}-1, t\right) 1_{i \notin R_{q}} .
$$

If $i \in R_{q}$ we get

$$
\begin{aligned}
& f_{i}\left(q_{i}+1, r_{i}, t\right)-\left(q_{i}+\rho_{i}\left(1-a_{i}+a_{i} h_{i}\left(r_{i}, t\right)\right)\right) f_{i}\left(q_{i}, r_{i}, t\right) \\
& +\rho_{i} q_{i} f_{i}\left(q_{i}-1, r_{i}, t\right)=0 .
\end{aligned}
$$

This is a recursion only for $q_{i}$, while $r_{i}$ and $t$ are only parameters. Lemma 4 gives the solution to (70). For $i \notin R_{q}$ in the policy of Example 2 we can satisfy (70) by assigning $q_{i}=C_{i}$. Here $h_{i}\left(r_{i}, t\right) \neq 0$ and thus (69) gives a recursion equation from which $g_{i}\left(r_{i}\right)$ is solved by the form

$$
\begin{gathered}
g_{i}\left(r_{i}, t\right)=g_{i}\left(r_{i}-1, t\right) \prod_{j=1}^{r_{i}} D_{i, r_{i}}, \\
D_{i, j}=\frac{\rho_{i} h_{i}(j, t) f_{i}\left(C_{i}, j-1, t\right)}{C_{i}+\rho_{i}\left(1-a_{i}+a_{i} h_{i}(j, t)\right) f_{i}\left(C_{i}, j, t\right)-\rho_{i} f_{i}\left(C_{i}-1, j, t\right)}
\end{gathered}
$$

where $C_{i}$ is the maximum value for $q_{i}$.

Let us look at the special case $h_{i}\left(r_{i}, t\right)=0$. Then $f_{i}\left(q_{i}, r_{i}, t\right)=f_{i}\left(r_{i}\right)$. Instead of (67) we will select

$$
s_{t, q_{i}, r_{i}}=\frac{1}{q_{i} !} f_{i}\left(q_{i}\right) g_{i}\left(r_{i}\right) t^{-r_{i}}
$$

and in (68)-(69)

$$
h_{i}\left(r_{i}\right) \frac{t^{-\left(r_{i}-1\right)}}{t^{-r_{i}}}=\frac{r_{i}}{a_{i} \lambda_{i}} \neq 0 .
$$

We get an equation similar to (69) and can solve $g_{i}\left(r_{i}\right)$. Scaling by selecting the parameter $A$ lets us satisfy

$$
\sum_{r, q} s_{n, q, r}=1 \text { and } 1 \geq s_{n, q, r} \geq 0 .
$$

There is still the requirement that the sum over $r$ of values $s_{n, q, r}$ must be finite. The part $s_{n, r}$ in $s_{n, q, r}$ satisfies it.

\section{APPENDIX III: ProOFS OF SECTION IV}

For notational simplicity, we write $\bar{q}$ simply as $q$.

Proof of Lemma 1: Since (23) is a special case of (28), it suffices to show the claim for (28) and (16). In (28) holds

$$
\begin{aligned}
& \lim _{n \rightarrow \infty} \sum_{r=0}^{\infty} r\left(a s_{n, r}(q) \Delta s_{n, q}+(1-a) \Delta v_{n, q, r}\right)=0 \text { because } \\
& \lim _{n \rightarrow \infty} \Delta s_{n, q}=\Delta \pi_{q}=0 \text { and } \lim _{n \rightarrow \infty} \Delta v_{n, q, r}=0 .
\end{aligned}
$$

Thus

$$
\sum_{r=0}^{\infty} r\left(s_{n+1, q, r}-s_{n, q, r}\right)=\frac{T}{N} \sum_{j \notin R_{q}} \lambda_{j} \omega_{j} .
$$

To prove the claim to (16), it is only needed to multiply (16) by $r$ and sum over $r$ 


$$
\sum_{r=0}^{\infty} r\left(s_{n+1, q, r}-s_{n, q, r}\right)=\frac{T}{N} \sum_{j \notin R_{q}} \lambda_{j} \omega_{j}
$$

Proof of Theorem 2: It is sufficient to show that the equations

$$
\begin{gathered}
\pi_{q_{1}, \ldots, q_{K}}=\lim _{n \rightarrow \infty} \sum_{r=0}^{\infty} s_{n, q_{1}, \ldots, q_{K}, r}, 0 \leq s_{n, q_{1}, \ldots, q_{K}, r} \leq 1, \\
s_{n+1, q_{1}, \ldots, q_{K}, r}=s_{n, q_{1}, \ldots, q_{K}, r} \\
-\frac{T}{N} \sum_{j \notin R_{q}} \lambda_{j} s_{n, q_{1}, \ldots, q_{K}, r}+\frac{T}{N} \sum_{j \notin R_{q}} \lambda_{j} s_{n, q_{1}, \ldots, q_{K}, r-\omega_{j}}
\end{gathered}
$$

are satisfied. Let us write

$s_{n, r_{1}, \ldots, r_{K}}(q)=$

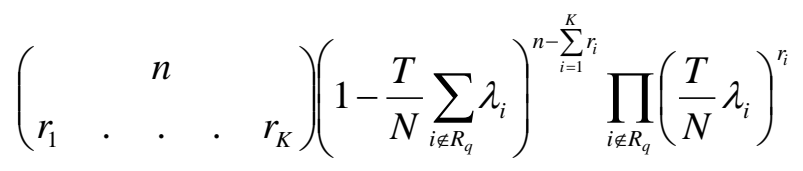

and re-index

$$
\left\{j \mid j \notin R_{q}\right\}=\left\{j_{1}, \ldots, j_{m}\right\}, i_{k}=r_{j_{k}}, \beta_{k}=\frac{T}{N} \lambda_{j_{k}} .
$$

Then

$$
\begin{aligned}
& s_{n, r_{1}, \ldots, r_{K}}(q)= \\
& \left(\begin{array}{ccccc} 
& & n & \\
i_{1} & . & . & . & i_{m}
\end{array}\right)\left(1-\sum_{k=1}^{m} \beta_{k}\right)^{n-\sum_{k=1}^{K} i_{k}} \prod_{k=1}^{m} \beta_{k}^{i_{k}}
\end{aligned}
$$

The basic multinomial recurrence relation (see e.g. [13])

$$
\begin{aligned}
& \left(\begin{array}{ccccc} 
& & n+1 & \\
i_{1} & \cdot & \cdot & \cdot & i_{m}
\end{array}\right)
\end{aligned}
$$

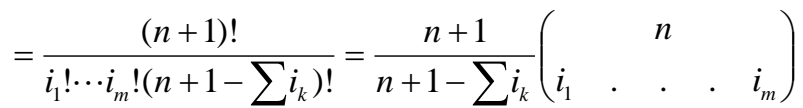

$$
\begin{aligned}
& =\left(\begin{array}{lllll} 
& n & \\
i_{1} & . & . & . & i_{m}
\end{array}\right)+\sum_{k=1}^{m}\left(\begin{array}{ccccc}
\multicolumn{4}{c}{n} \\
i_{1} & \ldots & i_{k}-1 & \ldots & i_{m}
\end{array}\right)
\end{aligned}
$$

shows that

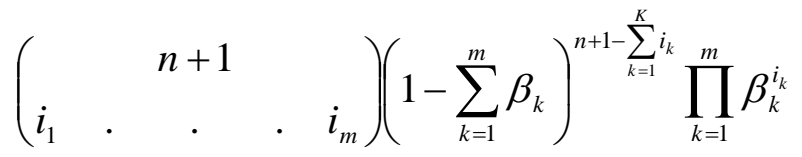

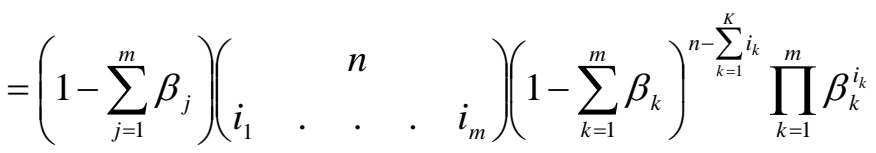

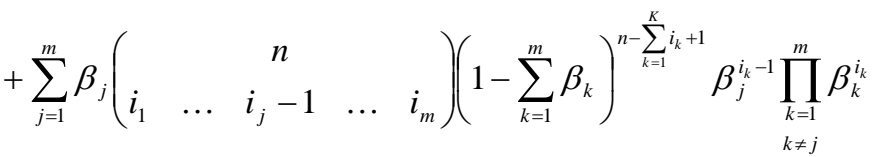

$$
\begin{aligned}
& s_{n+1, r_{1}, \ldots, r_{K}}(q) \\
& =s_{n, r_{1}, \ldots, r_{K}}(q)-\frac{T}{N} \sum_{j \notin R_{q}} \lambda_{j} s_{n, r_{1}, \ldots, r_{K}}(q)+\frac{T}{N} \sum_{j \notin R_{q}} \lambda_{j} s_{n, r_{1}, \ldots, r_{j}-1, \ldots, r_{K}}(q) .
\end{aligned}
$$

Summing over $A_{r, q}$ shows that the recursion equation is satisfied also for

$$
\begin{aligned}
& s_{n+1, q_{1}, \ldots, q_{K}, r}=\sum_{\substack{r_{1}=\ldots=r_{K}=0 \\
\left(r_{1}, \ldots, r_{K}\right) \in A_{r, q}}}^{n} s_{n+1, r_{1}, \ldots, r_{K}}(q) \pi_{n, q} \\
& =\left(1-\frac{T}{N} \sum_{i \notin R_{q}} \lambda_{i}\right) s_{n, q_{1}, \ldots, q_{K}, r}+\frac{T}{N} \sum_{i \notin R_{q}} \lambda_{i} \sum_{\substack{r_{1}=\ldots=r_{K}=0, r_{1}, \ldots, r_{i}-1, \ldots, r_{K} \\
\left(r_{1}, \ldots, r_{K}\right) \in A_{r, q}}}^{n} s_{(q) \pi_{n, q}} \\
& =\left(1-\frac{T}{N} \sum_{i \notin R_{q}} \lambda_{i}\right) s_{n, q_{1}, \ldots, q_{K}, r}+\frac{T}{N} \sum_{i \notin R_{q}} \lambda_{i} \sum_{\substack{r_{1}=\ldots, r_{i}^{\prime}=\ldots r_{k} \\
\left(r_{1}, \ldots, r_{i}^{\prime}, \ldots, r_{K}\right) \in r_{r, q-\omega_{j}}}}^{n} s_{n, \ldots, r_{i}^{\prime}, \ldots, r_{K}}(q) \pi_{n, q} \\
& =\left(1-\frac{T}{N} \sum_{i \notin R_{q}} \lambda_{i}\right) s_{n, q_{1}, \ldots, q_{K}, r}+\frac{T}{N} \sum_{i \notin R_{q}} \lambda_{i} s_{n, q_{1}, \ldots, q_{K}, r-\omega_{i}} . \\
& \sum_{r=0}^{\infty} s_{n, q_{1}, \ldots, q_{K}, r}
\end{aligned}
$$

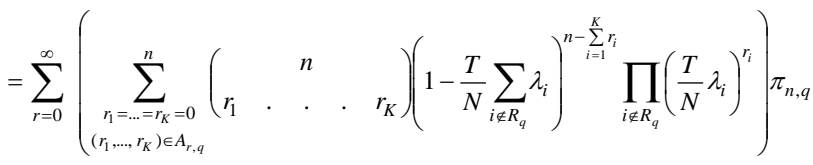

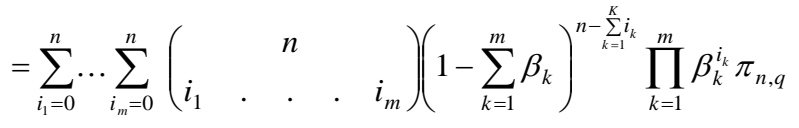

$$
\begin{aligned}
& =\pi_{n, q} \text {. }
\end{aligned}
$$

Clearly $0 \leq s_{n, q_{1}, \ldots, q_{K}, r} \leq 1$. This completes the proof.

Proof of Theorem 3: Write $\alpha_{k}=\frac{T}{N} \beta_{k}=\lambda_{j_{k}}$. The limit of the multinomial term

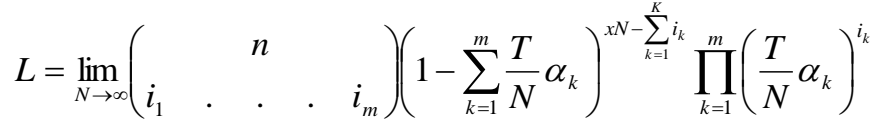

is calculated by Stirling's formula using Poisson's limit procedure:

$$
L=\prod_{i \notin R_{q}} \frac{\left(x T \lambda_{i}\right)^{r_{i}}}{r_{i} !} e^{-x T \sum_{i \notin R_{q}} \lambda_{i}} .
$$

Thus

Thus 


$$
s_{t, q_{1}, \ldots, q_{K}, r}=\lim _{N \rightarrow \infty} s_{x N, q_{1}, \ldots, q_{K}, r}=\sum_{\substack{r_{1}=\ldots=r_{K}=0 \\\left(r_{1}, \ldots, r_{K}\right) \in A_{r, q}}}^{n} \prod_{i \notin R_{q}} \frac{\left(x T \lambda_{i}\right)^{r_{i}}}{r_{i} !} e^{-x T \sum_{i \notin R_{q}} \lambda_{i}} \pi_{q}
$$

as claimed.

Proof of Lemma 2: Directly summing from (31) is difficult, but as (31) is the limit of (30), it can be calculated as $O\left(\frac{1}{N}\right)$ limit from (30). To get $O\left(\frac{1}{N}\right)$ limit, we only can take the terms $r_{i}=1, r_{j}=0$ for $i \neq j$, and $r_{1}=\ldots=r_{K}=0$ since there is the term $\left(\frac{T}{N}\right)^{r_{i}}$ in (30). The term $r_{1}=\ldots=r_{K}=0$ disappears as $r=0$ and for the other terms $r=\omega_{i}$. Consequently, from (30)

$$
\begin{aligned}
& \sum_{r=0}^{\infty} r s_{n, q_{1}, \ldots, q_{K}, r}=\sum_{i \notin R_{q}} \omega_{i} \lambda_{i} n\left(1-\frac{T}{N} \sum_{j \notin R_{q}} \lambda_{j}\right)^{n-1} \frac{T}{N} \lambda_{i} \pi_{q}+O\left(N^{-2}\right) \\
& =\sum_{i \notin R_{q}} \omega_{i} \lambda_{i} n \frac{T}{N} \lambda_{i} \pi_{q}+O\left(N^{-2}\right) .
\end{aligned}
$$

Thus

$$
\begin{aligned}
& \sum_{r=0}^{\infty} r s_{t, q_{1}, \ldots, q_{K}, r}=\lim _{N \rightarrow \infty} \sum_{r=0}^{\infty} r s_{x N, q_{1}, \ldots, q_{K}, r} \\
& =\lim _{N \rightarrow \infty} \sum_{i \notin R_{q}} \omega_{i} \lambda_{i} x T \pi_{q}=t \sum_{i \notin R_{q}} \omega_{i} \lambda_{i} \pi_{q} . \square
\end{aligned}
$$

\section{ACKNOWLEDGMENT}

Author is deeply grateful to Dr. Markku Liinaharja from Helsinki University of Technology (HUT) for reading the proofs - any possibly remaining errors are solely on author's responsibility, to Dr. Jouni Karvo, also from HUT, for suggesting improvements to the structure and readability of the paper, and to the anonymous reviewers for their helpful comments.

\section{REFERENCES}

[1] F. P. Kelly, "Charging and rate control for elastic traffic," Eur. Trans. Telecommun., vol. 8, pp. 3-37, 1997.

[2] F. P. Kelly, A. K. Maulloo, and D. K. H. Tan, "Rate control for communication networks: Shadow prices, proportional fairness and stability, “J. Oper. Res. Soc., vol. 49, pp. 237-252, 1998.

[3] R. J. Gibbens and F. P. Kelly, (1999) Resource pricing and the evolution of congestion control, [Online.]. Avaible: http//www.statslab.cam.ac.uk/ frank/evol.html

[4] H. Yaiche, R. R. Mazumdar, and C. Rosenberg, "A Game Theoretical Framework for Bandwidth Allocation and Pricing in Broadband Networks," IEEE/ACM Tr. on Networking, Vol. 8. No. 5, pp. 667678, 2000 .

[5] X.-R. Cau, H.-X. Shen, R. Milito, and P. Wirth, "Internet Pricing With Game Theoretical Approach, Concepts and Examples," IEEE/ACM Tr. on Networking, Vol. 10. No. 2, pp. 208-216, 2002.

[6] I. Ch. Paschalidis, and Y. Liu, "Pricing in Multiservice Loss Networks: Static Pricing, Asymptotic Optimality, and Demand Substitution Effects," IEEE/ACM Tr. on Networking, Vol. 10. No. 3, pp. 425-438, 2002.

[7] H. Rummukainen and J. Virtamo. "Polynomial Cost Approximations in Markov Decision Theory Based Call Admission Control," IEEE Trans. on Networking, Vol. 9, No 6, pp. 769-779, 2001,.
[8] K. W. Ross, Multiservice Loss Models for Broadband Telecommunication Networks, New York: Springer-Verlag, 1995.

[9] F. P. Kelly, Reversibility and Stochastic Networks, New York: Wiley, 1979.

[10] K. R. Krishnan and F. Hübner - Szabo de Bucs, "Admission control and state-dependent routing for multirate circuit-switched traffic," in Proc. $15^{\text {th }}$ ITC, V. Ramaswami and P. E. Wirth, Eds.: Elsevier Science B. V., pp. 1043-1055, 1997.

[11] Z. Dziong, K.-Q. Lio, L. Mason, and N. Tetreault, "Bandwidth management in ATM-networks," in Proc. $13^{\text {th }}$ ITC, A. Jensen and V. B. Iversen, Eds., pp. 821-827, 1991.

[12] P. Marbach and J. N. Tsitsiklis, "A neuro-dynamic programming approach to call admission control in integrated service networks: The single-link case, " MIT, Cambridge, MA., Techn. Rep. Lab. Inform. Decision Syst. Rep. LIDS-P-2402. [Online.]

Available: http://web.mit.edu/jnt/www/publ.html, Nov, 1997.

[13] J. Riordan, Combinatorial Identities, New York: Krieger, 1979.

[14] Y. Kogan, "Exact analysis for a class of simple, circuit-switched networks with blocking," Adv. Appl. Probabil., vol. 21, pp. 952-955, 1989.

[15] C. Y. Li, P. K. A. Wai, and V. O. K. Li, "The Decomposition of a Blocking Model for Connection-Oriented Networks," IEEE/ACM Tr. on Networking, Vol. 12. No. 3, pp. 549-558, June 2004.

[16] D. Mitra, and Q. Wang, "Stochastic Traffic Engineering for Demand Uncertainty and Risk-Aware Network Revenue Management," IEEE/ACM Tr. on Networking, Vol. 13. No. 2, pp. 221-233, 2005.

[17] T. K. Nayak, and K. N. Sivarajan, "Dimensioning Optical Networks Under Traffic Growth Models," IEEE/ACM Tr. on Networking, Vol. 11. No. 6, pp. 935-947, 2004.

[18] Y. d'Halluin, P. A. Foryth, and K. R. Vetzal, "Managing Capacity for Telecommunications Networks Under Uncertainty," IEEE/ACM Tr. on Networking, Vol. 10. No. 4, pp. 579-588, 2002.

Jorma Jormakka (M'98) received the Ph.D. degree in mathematics 1988 from the University of Helsinki.

$\mathrm{He}$ is a professor of military technology at the Department of Military Technology at the National Defence College of Finland, where his area includes command, control and communication systems. His research interests focus currently on C3 platforms, network-centric warfare, information warfare, service and management architectures, computer security and quality of service issues. Since 1987 Jormakka has worked in telecommunications in various academic and industrial research institutes . 